\title{
Imaging of Aluminum Nanoparticles Embedded in an Amorphous Sapphire Substrate Using Plasmon Energy-loss Electrons in TEM
}

\author{
Yaqiao $\mathrm{Wu}^{1,2}$ and Janet Callahan ${ }^{1}$ \\ ${ }^{1 .}$ Micron School of Materials Science and Engineering, Boise State University, Boise, USA. \\ 2. Center for Advanced Energy Studies, Idaho Falls, USA.
}

Nanoparticles may be formed through ion implantation of metal ions into an oxide substrate. Typically, this forms precipitates of the implanted ion; however if the ion selected for implantation is a reactive element, the oxide substrate may be reduced by the implant ion. In this case, nanoparticles comprised of the reduced substrate cation can coalesce to form nano-dimensional features [1]. In this work, yttrium ions were accelerated to energies of $100 \mathrm{keV}$ or $150 \mathrm{keV}$ and implanted into single crystal R-plane sapphire to fluences of $5 \times 10^{16} \mathrm{ions} / \mathrm{cm}^{2}$. This process produces nanoparticles embedded in an amorphous matrix that contains $\mathrm{Al}, \mathrm{Y}$ and $\mathrm{O}$. This work is focused on providing information about the particles and nano-features produced as a result of these treatments.

Cross-sectional TEM lamella was prepared by first sputter coating Pt on the sample surface, and then using the lift-out technique in a FEI Quanta 3D FEG FIB. The lamella was mounted on a Mo-grid. TEM was conducted using a FEI Tecnai $\mathrm{G}^{2}$ F30 STEM FEG capable of doing electron energy lose spectroscopy (EELS), energy filtered TEM (EFTEM) and energy dispersive X-ray spectroscopy (EDS).

The $150 \mathrm{keV}$ sample shows an amorphous layer that extends approximately $110 \mathrm{~nm}$ from the surface of the sample, see Fig. 1a. The BF-TEM image shows fine particles in the center of the layer. Corresponding diffraction patterns collected from regions of the layer that do not show particles reveal an amorphous diffraction pattern (Fig. 1b). The particles are crystalline as seen from the lattice fringes and inset CBEDP obtained from the particle (Fig. 1c). Immediately as the amorphous layer ends, lattice fringes from the sapphire matrix are evident (SAEDP of this region is shown as inset in Fig. 1a); there is some contrast resulting from residual ion beam damage that diminishes with depth into the substrate.

The $100 \mathrm{keV}$ sample is seen in Fig. 2a. The amorphous layer is $90 \mathrm{~nm}$ thick, and the particles have less contrast compared with Figure 1. Also, there is a band of contrast difference seen at the particles location (dashed-line-arrowed in Fig. 2a). High resolution imaging shows crystalline particles at location 2 which are 5 to $6 \mathrm{~nm}$ in diameter (Fig. 2b). The interface between the amorphous layer and the crystalline substrate is shown in Fig. 2 c.

Energy filtering was used to investigate the nanoparticulate features observed in both samples, see Figure 3. Using the zero-loss, $15 \mathrm{eV}, 25 \mathrm{eV}$ and $40 \mathrm{eV}$ energies, with a $5 \mathrm{eV}$ energy window, elemental maps were collected using EFTEM technique. The $15 \mathrm{eV}$ maps are shown in Figure 3. This energy level corresponds to the aluminium plasmon loss peak. Significantly more particles are seen, some of which have features that extend across one third or more of the amorphous later (compare Fig. 3a with $3 b$ ). Through comparison with the $25 \mathrm{eV}$ and $40 \mathrm{eV}$ maps, we conclude that the particles are composed of $\mathrm{Al}$, that they are oxygen deficient, and that they are not yttria. This finding is consistent with earlier work that was conducted in plan-view only [1].

References: 
[1] Hunt, E.M., Hampikian, J.M., Acta Materialia 47 (1999) p.1497.

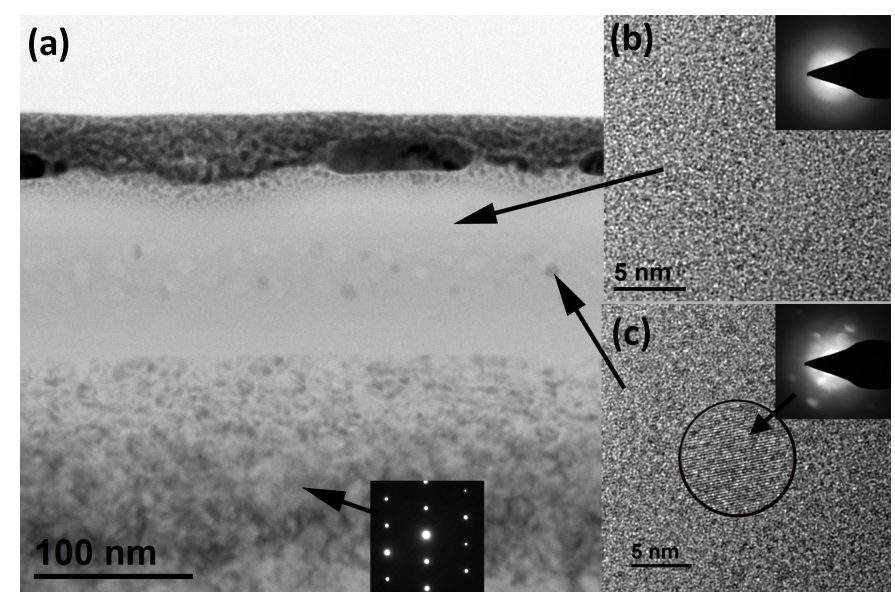

Figure 1. TEM images showing microstructure of sapphire surface experienced $150 \mathrm{keV}$ yttrium ion implantation. (a) surface structure, (b) amorphous layer and (c) nanocrystalline formed in the middle of amorphous layer.

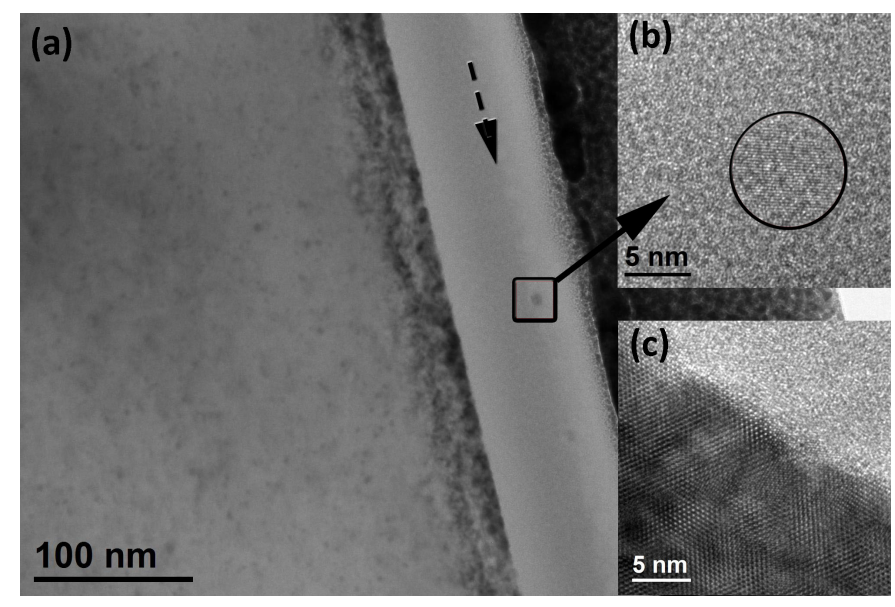

Figure 2. TEM images showing microstructure of sapphire surface experienced $100 \mathrm{keV}$ yttrium ion implantation. (a) surface structure, (b) nanocrystalline formed in the middle of amorphous layer and (c) interface between amorphous layer and substrate.

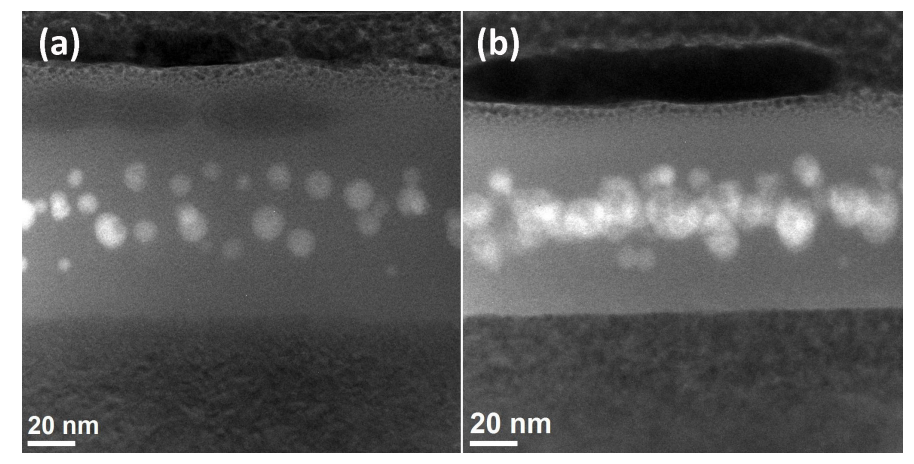

Figure 3. EFTEM elemental maps of (a) $150 \mathrm{keV}$ and (b) $100 \mathrm{keV}$ samples at $15 \mathrm{eV}$ energy, revealed the nanocrystallines in both samples are $\mathrm{Al}$. 